\title{
MAGREZA E SOBREPESO EM ESCOLARES DE RIO BRANCO, AC, BRASIL
}

\section{UNDERWEIGHT AND OVERWEIGHT IN SCHOOL CHILDREN FROM RIO BRANCO, ACRE STATE, BRAZIL}

\author{
Orivaldo Florencio de Souza1, Edson dos Santos Farias²
}

\begin{abstract}
RESUMO
Este estudo transversal investigou a prevalência de magreza e sobrepeso em escolares de Rio Branco, Acre, baseando-se nos padrões de crescimento infantil da OMS de 2007. Foram avaliados 706 escolares de 8 a 14 anos matriculados nas $3^{a}$ e $4^{a}$ séries de escolas públicas. Os escolares foram avaliados em faixas etárias de 8 a 9 anos, 10 a 11 anos e 12 a 14 anos. A prevalência geral de sobrepeso foi de $17,6 \%$ (17,3\% meninos e $17,8 \%$ meninas) e a magreza foi abaixo de $3,0 \%$, o que é um nível aceitável na população. Conclui-se que o sobrepeso está emergindo como um sério problema de saúde pública em escolares de ensino fundamental de escolas públicas da área urbana de Rio Branco, Acre.
\end{abstract}

Palavras-chave: estado nutricional; índice de massa corporal; criança.

\begin{abstract}
A cross-sectional study investigating the prevalence of underweight and overweight in school-aged children from Rio Branco, Acre State, based on the 2007 WHO growth reference, was carried out. A total of 706 children between 8 and 14 years of age, enrolled in public primary schools at $3^{\text {rd }}$ and $4^{\text {th }}$ grades were assessed. For the assessment, the school children were stratified into age bands of 8 to 9 years, 10 to 11 years, and 12 to 14 years. The overall prevalence of overweight was $17.6 \%$ ( $17.3 \%$ boys and $17.8 \%$ girls) while prevalence of underweight was less than 3.0\%, a level within normal limits for this population. The study results showed that overweight is becoming a major public health problem among children at state primary schools in urban areas of Rio Branco, Acre.
\end{abstract}

Key words: nutritional status; body mass index; child.

\section{INTRODUÇÃO}

As grandes mudanças no perfil nutricional da população têm se caracterizado pela redução na prevalência dos déficits nutricionais e ocorrências mais expressivas de sobrepeso em crianças, adolescentes e adultos. Esse processo é denominado transição nutricional. As causas estão relacionadas às mudanças no estilo de vida e padrões alimentares da população, inatividade física e determi-

1 Centro de Ciências da Saúde e do Desporto, Universidade Federal do Acre, Campus Universitário, BR 364, Km 04, Bairro Distrito Industrial, Rio Branco/AC.

2 Centro de Ciências da Saúde e do Desporto, Universidade Federal do Acre

Correspondência para: Luiz Ney (UFAC), Caixa Postal 2015, Rio Branco/AC, CEP 69.915-900 orivaldofs.ufac@gmail.com

Como citar este artigo:Underweight and overweight in school children from Rio Branco, Acre state, Brazil. Journal of Human Growth and Development 2011; 21(3): 878-882.

Artigo submetido em 16.03.11, aceito em 20.08.11. 
nada inter-relação dos fatores econômicos, demográficos e culturais ocorridos na sociedade ${ }^{1}$.

A desnutrição em crianças brasileiras até a década de 80 representava um dos maiores problemas de saúde públi$\mathrm{ca}^{2}$. Informações recentes da Pesquisa Nacional de Saúde Demografia de 2006 evidenciaram redução da prevalência da desnutrição em crianças brasileiras ${ }^{3}$. Porém, essa redução ocorreu de modo desigual entre as regiões brasileiras. Em contraste às demais regiões brasileiras, na Região Norte a desnutrição continua sendo um importante problema na saúde de crianças.

Atualmente, no Brasil, a prevalência de sobrepeso superou a prevalência de magreza ${ }^{4}$. O sobrepeso é um distúrbio nutricional que pode ter início em qualquer idade ${ }^{5,6}$ e apresenta características multifatoriais. Além disso, existem evidências do sobrepeso continuar da infância e adolescência para a fase adulta, elevando a morbi-mortalidade associadas ao excesso de peso e diminuindo a expectativa de vida?.

No município de Rio Branco, AC, as informações sobre magreza e sobrepeso em crianças e adolescentes são escassas ${ }^{8,9}$. Esse fato dificulta a implementação de políticas públicas de prevenção e intervenção sobre o estado nutricional em crianças e adolescentes em instituições escolares. Assim, o objetivo é identificar a prevalência de magreza e do sobrepeso em escolares das $3^{a}$ e $4^{a}$ séries do ensino fundamental da cidade de Rio Branco, AC.

\section{MÉTODO}

Trata-se de um estudo transversal de base escolar, realizado com crianças e adolescentes das $3^{\circ}$ e $4^{\circ}$ séries do ensino fundamental de escolas públicas da zona urbana de Rio Branco, Acre, no segundo semestre de 2004. O projeto desta pesquisa foi previamente aprovado pelo Comitê de Etica em Pesquisa da Fundação Hospital Estadual do Acre (01/2004).

Para determinação do tamanho mínimo da amostra foi adotada a preva- lência esperada de $18 \%$, conforme prevalência já identificada para o Brasil por Wang, Monteiro e Popkin ${ }^{4}$, e com a precisão fixada para um erro de amostragem em $4 \%$ ao nível de confiança de $95 \%$ e efeito de delineamento 2 para restringir o efeito do desenho amostral ${ }^{10}$. $\mathrm{O}$ tamanho mínimo da amostra foi de 708 escolares.

Na seleção dos escolares, utilizouse a amostragem conglomerada em um estágio, onde as unidades primárias foram as escolas. Do total das 70 escolas municipais e estaduais que ofertavam as $3^{\circ}$ e $4^{\circ}$ séries do ensino fundamental, foram sorteadas por amostragem aleatória simples 7 escolas (justificada por restrições operacionais, como por exemplo, número de pesquisadores e tempo disponível para a realização das entrevistas) para participarem do estudo. Todas as turmas de $3^{\circ}$ e $4^{\circ}$ séries do ensino fundamental de cada escola sorteada, totalizando 744 alunos, foram convidadas para participarem da coleta de dados. O critério de exclusão foi apresentar doenças em geral que impossibilitasse a mensuração antropométrica.

Para a mensuração do peso foi utilizada uma balança portátil digital eletrônica, com sensibilidade de 50 gramas e capacidade máxima de $150 \mathrm{~kg}$; a estatura foi mensurada por um estadiômetro portátil, subdividida em $0,1 \mathrm{~cm}$ e extensão máxima de 2 metros. Medidas de peso e estatura foram obtidas nas crianças durante o período de aula utilizando os procedimentos descritos por Lohman, Roche e Martorell ${ }^{11}$.

O escore $Z$ do índice de massa corporal pela idade (IMC/idade) foi obtido conforme as recomendações do novo referencial da Organização Mundial da Saúde $^{12}$ (OMS) de 2007. De acordo com os pontos de corte utilizados pelo referencial OMS de 2007, o IMC/idade foi classificado em magreza ( $<-2$ escore $Z$ ), sobrepeso (entre $>+1$ e $<+2$ escore $Z$ ) e obesidade ( $>+2$ escore $Z$ ). Para análise dos dados, as classificações de sobrepeso e obesidade foram agrupadas e denominadas em sobrepeso.

Os dados foram digitados no programa Excel $^{\circledR}$. Após esse processo, os 
dados foram transferidos para o programa estatístico Stata ${ }^{\mathrm{TM}} 9.2$ para análise estatística no módulo Survey. Na análise dos dados, foram calculados freqüências, prevalências e respectivos intervalos de confiança em $95 \%$.

\section{RESULTADOS}

Do total de 744 alunos identificados nas $3^{\circ}$ e $4^{\circ}$ séries do ensino fundamental de escolas públicas da zona urbana de Rio Branco, 8 alunos foram excluídos por problemas de saúde que impediam a mensuração antropométrica. Uma turma de 30 alunos foi também excluída por questões administrativas de uma escola. Assim, foram analisados 706 escolares de 8 a 14 anos, sendo $46,6 \%$ do sexo masculino e $53,4 \%$ do sexo feminino (Tabela 1). Os escores

Tabela 1: Distribuição dos escolares do ensino fundamental de Rio Branco, Acre - 2004.

\begin{tabular}{lcc}
\hline & $\mathbf{N}$ & $\mathbf{\%}$ \\
\hline Todos & 706 & 100,0 \\
Sexo & & \\
$\quad$ Masculino & 329 & 46,6 \\
$\quad$ Feminino & 377 & 53,4 \\
\hline Idade & & \\
$\quad 8-9$ anos & \\
$10-11$ anos & 387 & 40,6 \\
$12-14$ anos & 76 & 48,6 \\
\hline Índice de massa corporal & & 10,8 \\
$\quad$ Magreza & 18 & 2,5 \\
$\quad$ Eutrófico & 564 & 79,9 \\
$\quad$ Sobrepeso & 124 & 17,6 \\
\end{tabular}

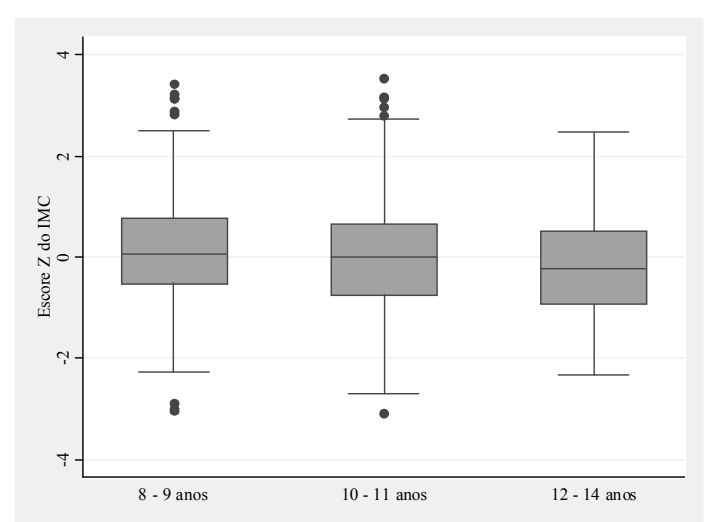

Figura 1: Distribuição do escore $Z$ do índice de massa corporal (IMC) por idade em escolares do ensino fundamental de Rio Branco, Acre - 2004.
$Z$ do índice IMC/idade dos escolares mostraram amplitude entre $-4,0$ e +4 (Figura 1).

De modo geral, houve prevalência alta de sobrepeso e baixa de magreza em todas as faixas etárias (Tabela 2). A prevalência de sobrepeso foi aproximada entre os sexos. Também foram identificadas menores prevalências de sobrepeso nas faixas etárias maiores. Apesar de o sexo feminino apresentar prevalência baixa de magreza $(3,2 \%)$, esse valor foi o dobro em contraste ao sexo masculino.

Tabela 2 - Prevalência de magreza, eutrófico e sobrepeso por sexo e faixa etária em escolares do ensino fundamental de Rio Branco, Acre - 2004.

\begin{tabular}{lccc}
\hline & $\begin{array}{c}\text { Magreza } \\
\%(\text { IC95\%) }\end{array}$ & $\begin{array}{c}\text { Eutrófico } \\
\%(\text { IC95\%) }\end{array}$ & $\begin{array}{c}\text { Sobrepeso } \\
\%(\text { IC95\%) }\end{array}$ \\
\hline $\begin{array}{l}\text { Sexo } \\
\quad \text { Masculino }\end{array}$ & $1,8(0,7 ; 4,1)$ & $80,9(76,5 ; 85,1)$ & $17,3(10,6 ; 27,0)$ \\
$\quad$ Feminino & $3,2(1,1 ; 8,4)$ & $79,0(74,9 ; 83,1)$ & $17,8(10,6 ; 28,1)$ \\
& & & \\
\hline Idade & & & \\
$\quad 8-9$ anos & $2,1(0,6 ; 6,5)$ & $78,4(73,6 ; 83,1)$ & $19,5(12,8 ; 28,5)$ \\
$10-11$ anos & $3,2(1,1 ; 8,5)$ & $79,8(75,6 ; 84,1)$ & $16,9(9,8 ; 27,5)$ \\
$12-14$ anos & $1,3(0,2 ; 6,4)$ & $85,5(77,5 ; 93,5)$ & $13,2(4,6 ; 31,8)$ \\
\hline
\end{tabular}

\section{DISCUSSÃO}

De acordo com o novo referencial da OMS de 2007, o sobrepeso foi identificado como um problema de saúde pública emergente que aflige crianças e adolescentes de 8 a 14 anos, de ambos os sexos, matriculados no ensino fundamental de escolas públicas da zona urbana de Rio Branco, Acre. Já a prevalência geral de magreza se enquadra na distribuição normal da população, estando abaixo de $3 \%$.

O referencial da OMS de 2007 foi utilizado por ser a atual recomendação para monitorar o crescimento e estado nutricional de crianças e adolescentes mundialmente. Enquanto a anterior recomendação do referencial do National Center for Health Statistics (NCHS) era baseada apenas em crianças e adolescentes dos Estados Unidos da América, o referencial da OMS de 2007 foi elabora- 
do a partir de dados coletados em seis países de vários continentes, inclusive o Brasil na América do Sul' ${ }^{13}$.

A comparação dos resultados do presente estudo usando o referencial da OMS de 2007 com dados de inquéritos nacionais e estudos regionais é limitada pelo uso de diferentes referenciais do estado nutricional. Entretanto, a prevalência de sobrepeso e de magreza observada em escolares de Rio Branco corrobora dados nacionais ${ }^{4}$ e do município de Porto Velho, Rondônia ${ }^{14}$, ambos utilizando o referencial do NCHS de 1977.

Informações atuais sobre magreza em crianças brasileiras com idade abaixo de 10 anos são escassas, o que limita a avaliação da sua gravidade. De qualquer forma, a prevalência de magreza identificada em escolares na faixa etária de 8-9 anos foi baixa, não representando um problema relevante de saúde pública. Além disso, essa prevalência também é baixa em comparação ao obtido por um inquérito nacional de 1997 que detectou prevalência de magreza em $7,7 \%$ (referencial do NCHS) das crianças e adolescentes brasileiros urbanos ${ }^{4}$. Contrastando com a prevalência de magreza revelada pela Pesquisa de Orçamentos Domiciliares ${ }^{9}$ de 2003/2004 para maiores de 10 anos de idade, também foram observados valores menores de prevalência de magreza nos escolares de Rio Branco.

A Pesquisa de Orçamentos Domiciliares ${ }^{9}$ aponta para a elevação da prevalência de excesso de peso em todas as regiões do Brasil. Na região Norte, o excesso de peso no sexo masculino aumentou substancialmente de 3,4\% para $16,2 \%$ no período de $1974 / 1975$ até $2003 / 2004$. No mesmo período e no sexo feminino, o aumento foi de 7,4\% para $13,2 \%$ de excesso de peso. Na classificação de magreza, dados da região Norte em ambos os sexos apontam que a classificação de magreza apresentou flutuações, com prevalência abaixo de $4,3 \%$. Estas informações sugerem a ocorrência da transição nutricional tanto no Brasil, como na região Norte.

Entretanto, no estado do Acre não há levantamentos em períodos anterio- res do IMC/idade em crianças e adolescentes que possibilite estabelecer uma série histórica. Contudo, existem indicativos que os escolares desse estudo estejam mais pesados do que os escolares de 1989. Conforme observações de Muniz ${ }^{8}$ no ano de $1989,2,2 \%$ dos escolares apresentavam índice peso para idade acima do percentil $97^{\circ}$ (referencial do NCHS), enquanto que no presente estudo a prevalência de sobrepeso foi de $17,6 \%$. A análise desse declínio é complexa, envolvendo aspectos sócio-econômicos e acesso dos escolares aos serviços de assistência à saúde, educação e saúde ambiental.

Evidências científicas informam que o sobrepeso em crianças pode continuar pela adolescência ${ }^{15,16}$ e ser desencadeador de doenças na vida adulta ${ }^{17}$. Outra evidência identificou que o sobrepeso em crianças e adolescentes já está associado às doenças crônicas não transmissíveis ${ }^{18}$. Nesse sentido, o estado de sobrepeso compromete a qualidade de vida e expõe os escolares de Rio Branco às doenças associadas ao sobrepeso.

Todos os valores de escore $Z$ do IMC por faixa etária e sexo mostraram amplitude abaixo de 4 unidades de escore $Z$. Desse modo, conforme recomendado pela OMS em 1997, não foram identificados valores implausíveis e as medidas antropométricas mostraram qualidade satisfatória para análise dos dados.

O desenho desse estudo foi elaborado para escolares das $3^{\circ}$ e $4^{\circ}$ séries. $A$ idade esperada para estas séries é de 8 até 11 anos. No entanto, é característico nas $3^{\circ}$ e $4^{\circ}$ séries do ensino fundamental haver alunos com idade acima de 11 anos. As causas do atraso escolar são diversas, como repetições por aprendizagem insuficiente ou histórico de doenças passadas que dificultava a aprendizagem ou impediam o aluno ir à escola.

Assim, nesse estudo foram apresentadas informações de que o sobrepeso consiste em um problema nutricional em escolares das $3^{\circ}$ e $4^{\circ}$ séries em Rio Bran$\mathrm{co}$, constituindo-se em indicador da necessidade imediata de ações para conter a evolução do sobrepeso nos escolares de Rio Branco, Acre. 


\section{REFERÊNCIAS}

1. Popkin BM. Contemparary nutrition transition: determinants of diet and its impact and its on body composition. Proc Nutr Soc. 2011;70:82-91.

2. Monteiro CA. Velhos e novos males no Brasil: a evolução do país e de suas doenças. $2^{\circ}$ ed. São Paulo: HUCITEC-NUPENS; 2000.

3. Monteiro CA, Benicio MHA, Konno SC, Silva ACF, Lima ALL, Conde WL. Causes for the decline in child undernutrition in Brazil, 1996-2007. Rev Saude Publica 2009; 43:35-43.

4. Wang Y, Monteiro CA, Popkin B. Trends of obesity and underweight in older children and adolescents in the United States, Brazil, China, and Russia. Am J Clin Nutr. 2002;75:971-77.

5. Silva MCP, Ramos CHB, Costa RF. Estado nutricional e níveis pressóricos de escolares adolescentes da cidade de Cubatão - SP, Brasil. Rev Bras Crescimento Desenvolv Hum. 2008; 18:288-97.

6. Simon VGN et al. Prevalência de sobrepeso e obesidade em crianças de dois a seis anos matriculadas em escolas particulares no município de São Paulo. Rev Bras Crescimento Desenvolv Hum. 2009;19:211-8.

7. Raj M, Kumar RK. Obesity in children and adolescents. J Med Res. 2010;132:598-607.

8. Muniz PT. Estado nutricional dos escolares de 7 a 18 anos da cidade de Rio Branco (Acre) e sua relação com alguns fatores sócio-econômicos. [Dissertação] Recife: Universidade Federal de Pernambuco; 1991.

9. Instituto Brasileiro de Geografia e Estatística. Pesquisa de Orçamentos Familiares - 2002-2003: antropometria e análise do estado nutricional de crianças e adolescentes do Brasil. Rio de Janeiro, RJ: IBGE; 2006.
10. Lwanga SK, Lemeshow S. Sample size determination in health studies. Geneva, Switzerland: WHO; 1991.

11. Lohman T, Roche A, Martorell R. Anthropometric standardization reference manual. Champaign, IL, USA: Human Kinetics; 1988.

12. Onis $M$, Onyango AW, Borghi $E$, Siyam A, Nishida C, Siekmann J. Development of a WHO growth reference for school-aged children and adolescents. Bull World Health Organ. 2007; 85:660-7.

13. Onis M, Garza C, Victora CG, Onyango AW, Frongillo EA, Martines J. The WHO Multicentre Growth Reference Study: planning, study design, and Methodology. Food Nutr Bull. 2004;25:S15-S26.

14. Farias ES, Guerra-Junior G, Petroski EL. Estado nutricional de escolares em Porto Velho, Rondônia. Rev Nutr. 2008;21:401-9.

15. Guo S, Chumlea W. Tracking of body mass index in children in relation to overweight in adulthood. Am J Clin Nutr. 1999;70:145S-8S.

16. Wang Y, GE K, Popkin B. Tracking of body mass index from childhood to adolescence: a 6-y follow-up study in China. Am J Clin Nutr. 2000;72:1018-24.

17. Barker DJ, Gluckman PD, Godfrey KM, Harding JE, Owens JA, Robinson JS. Fetal nutrition and cardiovascular disease in adult life. Lancet. 1993;341:938-41.

18. Katzmarzyk P, Srinivasan S, Chen W, Malina R, Bouchard C, Berenson G. Disease Risk Factors in a Biracial Sample of Children and Adolescents Body Mass Index, Waist Circumferen$\mathrm{ce}$, and Clustering of Cardiovascular. Pediatrics. 2004;114:198-205.

19. Ogden $C L$, Carroll MD, Flegal KM. High body mass index for age among US children and adolescents, 20032006. JAMA. 2008;299:2401-5. 